- levels are still on the rise," he says.

To meet the targets, "China must switch from a model of economic development at all costs to one that is much more sustainable", adds He. "This is a unique opportunity to make that transition."

But the switch will not be easy, says Ma Jun, director of the non-governmental Institute of Public and Environmental Affairs in Beijing. "The resistance from industry groups and some government agencies will be fierce because of vested interest." Ma adds that institutional reforms and amendments to environmental laws will be crucial to the scheme's success.

At the moment, the environment ministry is powerless to act against many polluters, which often ignore it or happily pay fines that have little effect on their profits. The ministry "is seriously under-resourced", says Michael Walsh, founding chair of the International Council on Clean Transportation, a non-profit organization headquartered in Washington DC. The Chinese ministry has, for instance, only a few dozen staff to safeguard air quality. By comparison, the US Environmental Protection Agency has more than 1,000, Walsh says.

Analysts expect the government to reorganize ministries substantially at the annual meeting of the National People's Congress next month. Measures are likely to include slimming down powerful agencies such as the National Development and Reform Commission and the Ministry of Land and Resources, and granting the environment ministry more power and resources. Also on the agenda are

"The resistance from industry groups and some government agencies will be fierce because of vested interest." amendments to the 1989 Environmental Protection Law and the 1987 Atmospheric Pollution Prevention Act. These are expected to allow the environment ministry to impose much heftier fines on heavy polluters, veto projects that fail to address environmental impacts and shut down persistent offenders.

Political issues aside, certain scientific questions must be resolved urgently to ensure that the targets for 2017 are met, researchers say. "Air pollution in China is extremely complicated," says Zhu Tong, an atmospheric chemist at Peking University in Beijing. Smog can be caused by vehicle emissions, the burning of coal and biomass, dust and rubbish incineration. "It's unclear how their relative contribution is different in each city and varies from season to season."

As China prepares to use economic incentives and legal measures to curb pollution, it is also "making encouraging strides in promoting transparency of pollution information", says Ma. Nearly 200 cities across the country currently release air-quality information in real time, and that is set to increase to all 338 major cities next year. The environment ministry publishes a monthly list of the most polluted cities, which can affect housing prices, hit tourism and investment and provoke public outcry. Since January, the ministry has also rolled out a national monitoring system that requires more than 15,000 heavy polluters to post on the Internet their real-time emissions and discharges into rivers.

"When the information is out in the open, the polluters will be forced to act," says Ma. "The public is the strongest ally in the battle against air pollution." -

\title{
ASTRONOMY
}

\section{Missing galaxy mass found}

\section{Gravitationallensing solves puzzle from the Big Bang's echo.}

\section{BY EUGENIE SAMUEL REICH}

$\mathrm{S}$ oon after the Big Bang, there were tiny ripples: quantum fluctuations in the density of the seething ball of hot plasma. Billions of years later, those seeds have grown into galaxy clusters - sprawling groups of hundreds or thousands of galaxies bound together by gravity.

But there seems to be a mismatch. Results released last year suggest that as much as $40 \%$ of galaxy-cluster mass is missing when compared with the amount of clustering predicted by the ripples ${ }^{1}$. The findings have led theorists to propose physics beyond the standard model of cosmology to make up the difference. But a reconciliation could be in the offing, using improved measurements of the cluster masses.

The mismatch was first detected by the European Space Agency's Planck spacecraft, which measured the fluctuations imprinted on the cosmic microwave background radiation left over from the Big Bang and compared them with clusters that it could see. "A lot of us are intrigued" by the discrepancy, says David Spergel, an astrophysicist at Princeton University in New Jersey, who studied the cosmic microwave background with Planck's predecessor, NASA's Wilkinson Microwave Anisotropy Probe. "There's something missing in our understanding."

Some theorists have played with the characteristics of neutrinos - ghostly, nearly massless subatomic particles - as a way of compensating. On 6 February, for example, physicist Wayne Hu of the University of Chicago in Illinois and his colleagues published ${ }^{2}$ a theory that the mismatch could be bridged if the three known types of neutrino were significantly heavier than thought, or if there were a fourth, as yet undiscovered species of neutrino. The extra neutrino mass would have had an effect on the growth of the primordial ripples, evening them out and resulting in fewer clusters being observed today.

Now two studies, one in preparation and one posted on the arXiv preprint server on 11 February $^{3}$, suggest that clusters actually have more mass than Planck estimated - and thus
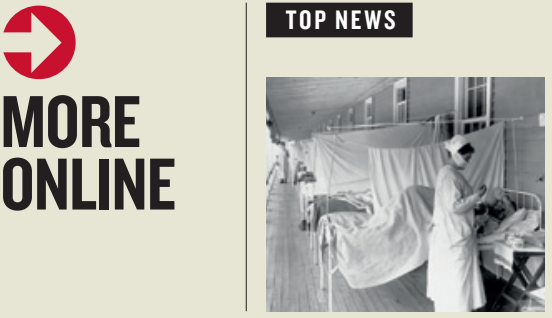

Spanish flu probably came from birds, not pigs go.nature.com/ xfjhip

\section{MORE NEWS}

- First results from portable gene sequencer go public go.nature.com/qvil3bc - PET scans trace spread of HIV analogue in macaques go.nature.com/ n8iz9m

- Extant genomes yield world atlas of human admixture go.nature.com/4twiy7

\section{NATURE PODCAST}

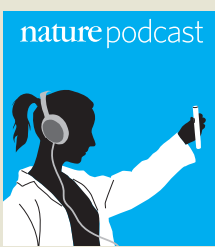

Pathogens in bees; the Nicaragua Canal; and news from the AAAS meeting nature. com/nature/podcast 


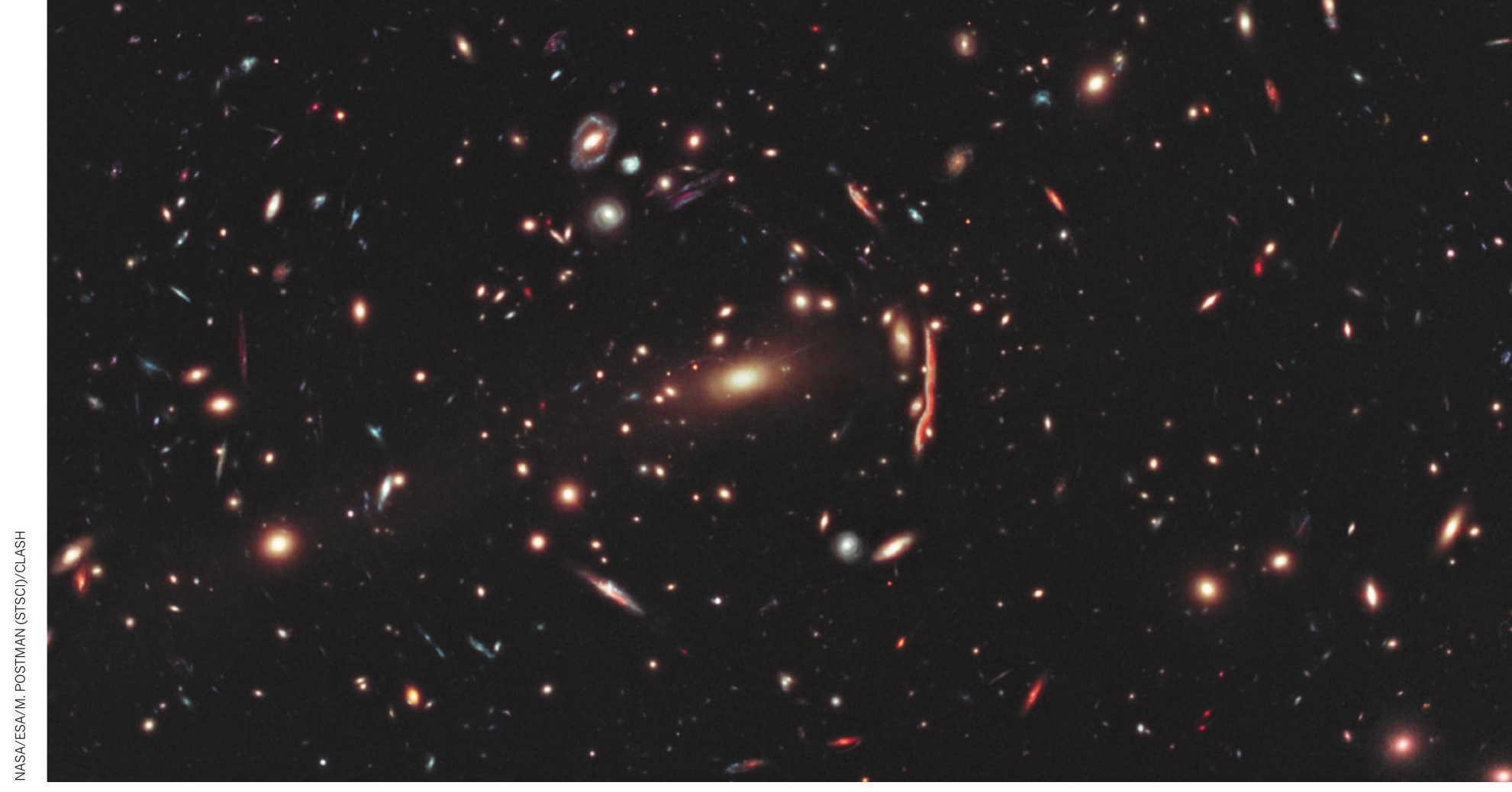

Galaxy clusters formed as a results of ripples in the very early Universe.

that there is little need for exotic physics. Both studies used gravitational lensing, a technique that weighs clusters by measuring how much their gravitational fields distort light that passes through them. "We think there's no problem," says Anja von der Linden, an astrophysicist at the Kavli Institute for Particle Astrophysics and Cosmology at Stanford University in California.

Von der Linden works on a project called Weighing the Giants, which used the Subaru telescope and the Canada-France-Hawaii telescope, both on Mauna Kea in Hawaii, to study 22 galaxy clusters also measured by Planck. It came up with an average cluster mass of $10^{15}$ solar masses, or about 1,000 times the mass of the Milky Way - an average that was $43 \%$ higher than Planck's estimates ${ }^{3}$. The other study, called the Cluster Lensing and Supernova Survey with Hubble (CLASH), used the Hubble Space Telescope to measure 25 clusters measured by Planck, and produced an estimate that was about $30 \%$ higher than Planck's.

The differences seem to be attributable to the uncertain nature of Planck's estimates, which rely on a process called the SunyaevZel'dovich effect (see 'Weighing up galaxy clusters'). Planck detects photons from the cosmic microwave background. On their way to the satellite, some of these microwaves pass through galaxy clusters. There, they encounter energetic electrons associated with clouds of hot gas. When the photons collide with the electrons, they are boosted to higher energies.

The strength of that signal can be correlated

\section{WEIGHING UP GALAXY CLUSTERS}

Using gravitational lensing to estimate the masses of galaxy clusters, astronomers think they can account for mass that seemed to be missing in estimates using the Sunyaev-Zel'dovich effect.

\section{The Sunyaev-Zel'dovich effect}

Cosmic microwave background radiation

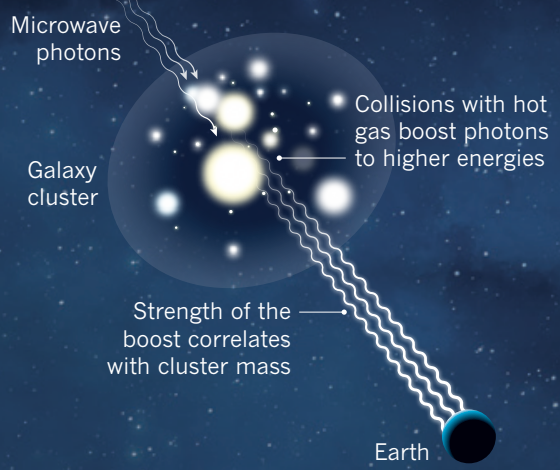

Gravitational lensing

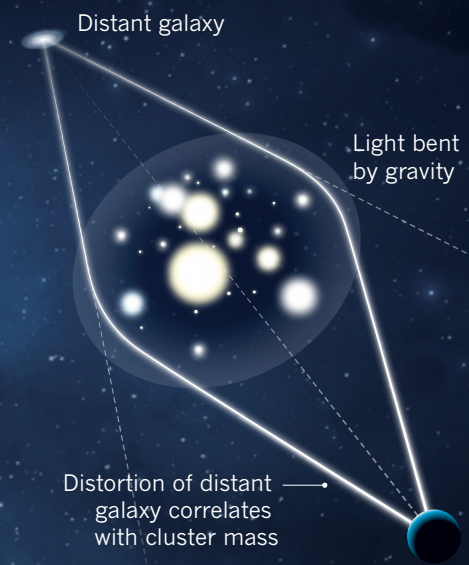

with the mass of all the galaxies in the cluster, because a larger cluster will trap more hot gas. But it is not a precise relationship. "That's the biggest source of uncertainty," says James Bartlett, a cosmologist at the University of
"There's something missing in our understanding." Paris Diderot who is part of the Planck collaboration. He says that Planck will release an updated analysis later this year that fine-tunes its mass calibration, and may bump up the cluster masses.

Many astrophysicists think that the remaining discrepancies will be resolved by data from other lensing surveys that are now starting up. The US\$50-million Dark Energy Survey, an optical-survey telescope at Cerro Tololo in Chile, completed its first three months of observations on 9 February. It measured hundreds of clusters and its first science results are expected later this year. Next month, a \$50-million Japanese instrument, Hyper Suprime-Cam, will be used to start a large lensing survey on the Subaru telescope.

Bradford Benson, an astrophysicist at the Fermi National Accelerator Laboratory in Batavia, Illinois, says that even if turns out that no cluster mass was missing after all, it will still be important to trace the evolution of today's clusters from the original cosmic ripples to see how the effects of dark energy, a mysterious repulsive force, may have changed with time. "Powerful data sets will be the next chapter in the story," he says.

1. Ade, P. A. R. et al. Preprint at http://arxiv.org/ abs/1303.5080 (2013).

2. Wyman, M., Rudd, D. H., Vanderveld, R. A. \& Hu, W. Phys. Rev. Lett. 112, 051302 (2014).

3. von der Linden, A. et al. Preprint at http://arxiv.org/ $\mathrm{abs} / 1402.2670$ (2014). 